\title{
¿Las condiciones socioeconómicas tienen efectos en el desempeño económico? El caso de la región del altiplano de San Luis Potosí en México
}

\author{
Do the socio-economic conditions have impact on the economic performance? \\ The case of the Altiplano region of San Luis Potosí in Mexico
}

\author{
Juan Carlos Yáñez-Luna*, Leonardo Tenorio Martínez, Leobardo Plata Pérez \\ Universidad Autónoma de San Luis Potosí, México
}

\begin{abstract}
Recibido el 14 de enero de 2019; aceptado el 4 de marzo de 2020
\end{abstract}
Disponible en Internet el: 18 de marzo de 2020

\section{Resumen}

El término "capital humano" se refiere al "nivel de educación" de una población, estas habilidades producirán valor económico, el cual tendrá un impacto importante en el desarrollo y crecimiento de una región. Esta investigación tiene como objetivo probar que las relaciones de algunas variables de condición social influyen en el desarrollo económico de una región determinada. El estudio se centra en la zona del Altiplano del Estado de San Luis Potosí en México. Se evaluaron datos demográficos de diversas localidades de la zona. Se utilizó el método de Mínimos Cuadrados Parciales (PLS-SEM) para estimar las relaciones causales entre variables como Educación y Salud con respecto al desarrollo económico y la migración en el contexto de estudio. Los resultados indican que un problema importante vinculado a la escasez de capital humano en esta región es la migración, que a su vez tiene efectos sobre el crecimiento económico y del desarrollo.

Código JEL: O1, O15, O18

Palabras clave: Crecimiento económico; Situación socioeconómica; Estudio subregional; Partial Least Squares

\footnotetext{
*Autor para correspondencia Correo electrónico: jcyl@uaslp.mx (J.C. Yáñez Luna).

La revisión por pares es responsabilidad de la Universidad Nacional Autónoma de México. 


\begin{abstract}
"Human capital" is a term that refers to the "level of education" of a population, these skills will produce economic value, which will have an important impact on the development and growth of a region. This research aims to prove that the relationships of some variables of social condition influence the economic development of a given region. The study focuses on the Altiplano area of the State of San Luis Potosí in Mexico. Demographic data from various localities in the area were evaluated. The method of Partial Least Squares (PLS-SEM) was used to estimate the causal relationships between variables such as Education and Health with respect to economic development and migration in the study context. The results indicate that a major problem linked to the scarcity of human capital in this region is migration, which in turn has effects on economic growth and development.
\end{abstract}

JEL Code: $\mathrm{O} 1, \mathrm{O} 15, \mathrm{O} 18$

Keywords: Economic growth; Socio-economic situation; Subregional study; Partial Least Squares

\title{
Introducción
}

Es conocida, desde los trabajos de Schultz (1960) y (Becker, 1964), la idea de capital humano, misma que reconoce la relación entre la educación y la capacitación con los mejores rendimientos económicos; mientras que recientemente la salud se ha reconocido como componente integral del capital humano (T. P. Schultz, 1997). Organismos internacionales como la UNESCO o la OCDE, con base en esa teoría, han logrado probar que las condiciones socioeconómicas en las que se desenvuelven los individuos tienen efectos sobre el desempeño educativo, tanto general como de las entidades y subregiones, lo que explica las diferencias tanto en términos de formación de capital humano como de los resultados económicos y de desempeño y crecimiento y desarrollo regional.

Es en este sentido que el presente trabajo busca probar la existencia de una relación entre las condiciones socioeconómicas y de educación con el desempeño económico en la región del altiplano del estado de San Luis Potosí (SLP), para lo cual se utilizaron los datos disponibles a nivel de localidades que para el año de $2010^{1}$ se tienen, con objeto de establecer un modelo que a la par de cumplir con las exigencias de los mínimos cuadrados parciales o $\mathrm{MCP}^{2}$, quien permite establecer relaciones estadísticamente fiables, nos ayude a explicar cómo influyen ciertas variables socioeconómicas a las educativas y éstas al desempeño económico en la región.

Este documento consta de tres por apartados. En el primero se realiza una revisión teórica

Datos obtenidos del censo de población y vivienda 2010.

${ }^{2}$ Esta herramienta de análisis se conoce en inglés como: Partial Least Squares (PLS). 
sobre cómo el desarrollo económico está explicado por condiciones sociales y educativas, así como una breve revisión de las condiciones que en este sentido prevalecen en la región del altiplano potosino. En los siguientes dos apartados se plantea el modelo y la metodología con que se abordó el problema. El análisis del modelo se realizó con base en la técnica estadística de análisis de rutas (Wright, 1934), la que permite al investigador conocer las relaciones causales que existe entre diversas variables, a su vez, para la evaluación y medición del modelo se utilizó la técnica de Mínimos Cuadrados Parciales (PLS). Así, la técnica por PLS “es la especificación de las relaciones causales y / o predictivas en términos de predictores (expectativas condicionales), seguida de la estimación por mínimos cuadrados de las incógnitas" (Wold, 1975), nos permitirán proponer algunas conclusiones finales.

\section{Situación educativa, desempeño económico y regiones}

La economía de la educación surge primero como tema de estudio para poder explicar aquella parte del crecimiento económico que no era posible explicar por los factores productivos. Con los trabajos seminales de (T. W. Schultz, 1961) nace primero la idea de capital humano y después que éste es resultado de la inversión futura en educación, de la que se desprenden dos condiciones: la eficiencia y la equidad.

De la condición de eficiencia se extrae el grado de rendimientos que la educación genera y que se miden en términos de beneficios económicos y/o sociales, en tanto que de la condición de equidad sea hace evidente la existencia de grados de aprendizajes producto de las diferentes condiciones en las que actúan los actores de la educación, por lo que para tener resultados homogéneos es necesario que el Estado ejerza una participación e inversión que permita alcanzarlos (Leyva López \& Cárdenas Almagro, 2002) de forma más amplia y generaliza, con el fin de limitar las desigualdades que por regiones pueden existir, producto de las condiciones histórico-económicas y con ello que los beneficios que resultan de éstas se distribuyan de forma más homogénea en la sociedad.

A partir de estas ideas, es factible pensar que la educación juega un papel medular en la mejora de las oportunidades de las personas, las regiones y los países, pero depende también directamente tanto de la estructura del sistema educacional regional como de los niveles de educación que los niños y jóvenes alcanzan, sus capacidades de aprovechamiento, quienes a su vez dependen de las condiciones socioeconómicas como: ruralidad, familia y cultura, mercado laboral, género, entre las más sobresalientes.

A partir del Tercer Estudio Regional Comparativo y Explicativo (TERCE) realizado por UNESCO, se pueden extraer conclusiones sobre la fuerte relación que existe entre educación y desempeño económico y cómo las condiciones en las que los niños y jóvenes aprenden tienen efectos fuertes y directos sobre los resultados educativos. Para 15 países de América 
Latina, este estudio dice que el nivel socioeconómico es la variable con mayor incidencia en el aprendizaje y que hay una relación directa entre desigualdad de la región y sus sistemas escolares (Treviño et al., 2016), lo que redunda en una baja movilidad social y se traduce en una trasmisión intergeneracional de la posición social y de pobreza (Romero Rodríguez, S.I., 2010).

Dentro de los hallazgos se encuentra el que la pobreza está asociada con la desigualdad lo que impide que se logren los objetivos educativos, ya que la pobreza genera restricciones en términos de alimentación y nutrición, medios básicos en vivienda y servicios y bienestar en general, lo que genera costos de oportunidad entre enviar o no a los niños y niñas al escuela, donde las niñas pueden estar en desventaja más clara (Treviño et al., 2016) ${ }^{3}$.

Como uno de los elementos principales para explicar el nivel de aprendizaje es el socioeconómico -quien considera variables al nivel de estudios de la madre y su tipo de empleo, nivel de ingresos, material del piso de la vivienda, servicios con los que cuenta incluyendo disponibilidad de libros- el estudio, para el caso mexicano, marca que el nivel de aprendizaje es considerablemente más alto si también lo es el ámbito social, lo que confirma la aseveración del estudio (Treviño et al., 2016).

En el estudio, la ruralidad, quien también puede explicar el aprovechamiento escolar al estar estrechamente relacionada con la pobreza, la desigualdad e iniquidad, muestra para México, que en promedio las poblaciones rurales presentan los menores niveles de desarrollo socioeconómico, mientras que las urbanas privadas presentan los mayores (Treviño et al., 2016).

La asistencia escolar, que puede estar sujeta al cumplimiento de ciertos requisitos, a procesos de selección escolar o a la necesidad de trabajar, también son evidenciados en el estudio como elementos que afectan el desempeño educativo y que provocan que la educación se trunque, por lo que se recomiendan políticas como la erradicación de cuotas obligatorias, procesos de selección y apoyos para que los niños no dejen de estudiar por trabajar (Treviño et al., 2016).

Al respecto, la Nueva Geografía Económica (NGE), al referirse a la relación entre educación, trabajo y remuneraciones, dice que las regiones que guardan distancias considerables entre los mercados de consumo y las fuentes de los insumos no pueden lograr la convergencia en niveles de ingreso y esto provoca que emerjan áreas económicas heterogéneas, además de que esas localidades que están alejadas de los centros económicos se caracterizan por tener bajos nivel educativos (López-Rodríguez et al., 2007), lo que puede estar condicionado por los procesos de liberalización comercial, quienes pueden estar permitiendo que ciertas regiones de países puedan integrarse de manera exitosa al comercio y/o los negocios internacionales, pero otras no. El caso de la migración será un fenómeno muy importante porque aquellos que puedan migrar hacia las regiones con mayores oportunidades de crecimiento económico lo

\footnotetext{
${ }^{3}$ De acuerdo con este estudio, México ha venido aumentando el porcentaje de población por debajo de la línea de pobreza, al pasar de 31.7 al 41.2 de 2006 a 2014.
} 
harán (Garduño Rivera, 2014), determinando niveles de crecimiento y productividad regional diferenciados (Kaldor, 1984).

Asimismo, la NGE sostiene que el tamaño de mercado, los costos de transportación, el comercio internacional y las economías de escala serán los principales determinantes de la concentración de actividades (Krugman \& Elizondo, 1996); (Fujita et al., 1999), lo que generará y explicará los niveles de heterogeneidad estructural tanto del aparato productivo y económico como del desempeño educativo y social.

\section{La región del Altiplano de San Luis Potosí}

En México desde finales del siglo pasado ya había un reconocimiento del impacto que tiene el crecimiento económico en el desarrollo nacional y regional (PND 1995-2000)4 ${ }^{4}$, pero desde entonces la heterogeneidad en las condiciones socioeconómicas regionales poco ha cambiado, lo que es evidente con el gran número de documentos que señalan los problemas de pobreza, educación, migración, salud, nutrición, ingresos, entre otros, relacionados a las condiciones socioeconómicas con evidente incidencia en el crecimiento y desarrollo económico regional (González Rivas, 2007; Mayer-Foulkes, 2008; Pintor Sandoval et al., 2017).

De acuerdo con el Índice de Competitividad Estatal 2016 del Instituto Mexicano de la Competitividad (IMCO), son seis los factores que permiten aumentar la competitividad de los estados que conforman al país: economía conectada con el exterior a través de exportaciones e Inversión Extrajera Directa (IED); alta actividad en la industria manufacturera; conexión a redes de energéticos; altos porcentajes de personal ocupado en empresas formales y salarios competitivos; mayor densidad de empresas grades que generan empleos formales y de calidad y sistemas de educación que generan capital humano y alineado a la estructura productiva del estado (Instituto Mexicano para la Competitividad, 2016).

Al respecto, San Luis Potosí ha mantenido en las dos últimas observaciones del índice el lugar 19, aunque en 2008 ocupó el 18, lo que es resultado de las mejoras en IED en los sectores automotriz y de autopartes principalmente, lo que genera mejoras en los rubros de comercio exterior, inversión de grandes empresas, formalidad y empleos con salarios bien remunerados, así como en su participación en el sector manufacturero. En este sentido su crecimiento económico ha estado cercano en el promedio nacional, pero altamente explicado por la subregión centro del estado.

Con base en el mismo estudio, los aspectos donde la entidad tiene áreas de oportunidad son los relativos al abastecimiento de fuentes energéticas de gas natural, el que es un factor determinante para el aumento de las inversiones y de la instalación de grandes empresas

\footnotetext{
${ }_{4}^{4}$ Plan Nacional de Desarrollo 1995-2000. Publicado por el Diario Oficial de la Federación. Disponible en: http://www.diputados. gob.mx/LeyesBiblio/compila/pnd.htm Consultado 12/10/2018
} 
nacionales e internacionales, pero que no ha sido resuelto satisfactoriamente. El rubro de inversiones en infraestructura física de calidad, transporte, vías de comunicación y carreteras, también se han estancado o no ha crecido con la celeridad necesaria, lo que la competitividad en términos de costos de transporte y traslado de personas y materiales.

En el ámbito educativo, es otro rubro con amplias áreas de oportunidad, ya que para el 2010 ningún municipio sobresalía entre aquellos con los promedios más altos de educación y de hecho el promedio escolar del estado es de 8.58 años cursados, mientras que el promedio nacional es de 8.63 años.

La relación entre educación e innovación se establece porque la concentración de fuerza de trabajo calificada en una región le permite a las empresas la adopción y desarrollo de nuevas tecnologías (Simón Fernández et al., 2004), lo que está siendo posible en el estado, dado el nivel de inversión para el desarrollo del capital humano que ha alcanzado. Específicamente con las becas para estudios de posgrado otorgadas a estudiantes del estado, la que reporta la primera posición a nivel nacional; así como las licenciaturas certificadas por el COPAES que representan el $2 \%$ en este mismo nivel y los siete programas de posgrado PNPC. Lo hecho en términos de términos de legislación de Ciencias y Tecnología y el que tenga grandes empresas que invierten en I+D en sus procesos productivos (Foro Consultivo Científico y Tecnológico, 2012), son factores que hablan en favor del estado.

La anterior información es producto del Ranking Nacional de Ciencia, Tecnología e Innovación (CTI) del Foro Consultivo científico y Tecnológico (FCCyT) el que busca tener una medida de comparación de los recursos que poseen cada estado del país y que ubica a SLP en el lugar 13 con un valor de -0.067 y que significa que el estado se encuentra muy cerca del promedio nacional, aunque por debajo.

Entre los componentes de este ranking está la infraestructura para la investigación, la productividad científica, población con estudios profesionales y de posgrado, entorno económico y social, TICS y formadores de recursos humanos, los que estarían explicando por qué el estado no logra saltar a los primeros lugares de este ranking o del de competitividad.

Este estudio señala como elementos en falta a las patentes otorgadas, la población con estudios de posgrado, el promedio de años estudiados, que ya señalábamos, el analfabetismo, baja conectividad de internet, así como de personal docente de posgrado y licenciatura, aunque en términos de investigadores miembros del Sistema nacional de Investigadores (SNI), la entidad ocupa la octava posición, lo que se considera un punto a favor (Foro Consultivo Científico y Tecnológico, 2012).

De acuerdo con una histórica división regional, SLP se divide en cuatro grandes regiones: Centro, Zona Media, Huasteca y Altiplano.

Nuestra región de estudio, el Altiplano, se encuentra ubicada al noreste del Estado y está compuesta por 15 municipios a saber: Catorce, Cedral, Charcas, Guadalcázar, Matehuala, 
Moctezuma, Salinas, Santo Domingo, Vanegas, Venado, Villa de Arista, Villa de Guadalupe, Villa de la Paz, Villa de Ramos y Villa Hidalgo.

El municipio de Matehuala es considerado el centro económico de la región, por desarrollar las más importantes actividades de la región y contener a las principales empresas de ésta, incluido al parque industrial de Matehuala.

El clima que va de lo desértico a lo semidesértico, en la parte norte de la región, pero en el que también se pueden encontrar vegetación de pino y encino, en la cercana a la capital del estado, permite como actividades económicas principales a la minería, el comercio, la agricultura protegida y la industria alimentaria y el turismo, lo que explica, al menos en parte, porque ocupa el segundo lugar del estado en cuanto a participación del PIB con un 6.4\%, lejos del primer lugar que ocupa la región Centro con un $84.1 \%$, quien por su parte desarrolla actividades económicas y de comercio internacional en los sectores: automotriz, metalmecánica, electrodomésticos y de servicios (Comité de Planeación del Desarrollo Estatal, 2016).

Con datos de 2015, el altiplano también ocupa el segundo lugar del PIB per cápita con \$59, 130 pesos, sin embargo, esta zona ocupa la tercera posición con relación a nivel de población, lo que hace de esta región muy importante de acuerdo con su capacidad productiva. En este mismo documento podemos encontrar que la región tiene alta participación en la minería, en especial en la producción de fluorita, cobre, yeso, oro, zinc y plata; la producción de carne en canal bovina y caprina y en el sector turismo esta ha venido aumentando su ocupación hotelera al 6\% de 2012 al 2014 (Comité de Planeación del Desarrollo Estatal, 2016), principalmente por las actividades económicas de Matehuala y el pueblo mágico de Real de Catorce.

\section{El modelo}

El modelo nos permitió explicar al ámbito económico de la región del altiplano de San Luis Potosí (SLP) con las siguientes variables: Economía, Educación Básica, Educación Media y Superior, Marginación, Migración y Salud. Estas variables fueron seleccionadas a partir de la información disponible en el Censo Nacional de Población y Vivienda $2010^{5}$ para San Luis Potosí, México, lo que generó que ciertas localidades, que no contenían información relevante para las variables mencionadas fueran eliminadas del estudio.

Bajo esta premisa se desarrolló un modelo basado en análisis de rutas para calcular la relación entre cada variable y su aportación hacia el desarrollo sustentable de la región. El método para la medición del modelo se realizará mediante la técnica de Mínimos Cuadrados Parciales para los modelos basados en análisis de Rutas (Wold, 1975). El modelo se centra en representar el Desarrollo Económico en dos aspectos: El primero relaciona el desarrollo

\footnotetext{
${ }^{5}$ El Censo Nacional de Población y Vivienda lo lleva a cabo el Instituto Nacional de Estadística, Geografía e Informática (INEGI), entre otros estudios de connotación económica y social.
} 
económico y la marginación social con el acceso de la población a los servicios de salud y de educación (nivel básico, medio y superior). Como ya se describió en el apartado anterior, el altiplano potosino suele ser una de las regiones con mayor índice de pobreza en el estado ${ }^{6}$ (De la Torre García \& Rodríguez García, 2014) por lo que consideramos que los accesos reducidos a los servicios de salud afectarán significativamente a sector educativo de la población y a su vez generará una mayor marginación social; es decir, si existe un determinado número de población en la región estudiada que no tiene accesos a los servicios básicos de salud, es muy probable que su desempeño y desenvolvimiento educacional se vea afectado negativamente. En este sentido, según Camberos \& Bracamontes (2007) la marginación se puede considerar como:

"una situación en la que viven dentro de una localidad o municipio, urbano o rural, un conjunto de individuos y familias que no satisfacen las necesidades consideradas básicas, de acuerdo con los criterios determinados por instituciones reconocidas como el Programa de las Naciones Unidas para el Desarrollo (PNUD) y el Banco Mundial”.

Así mismo los autores señalan que: "Por población marginada también se entiende al sector de la sociedad que por causas de la organización socioeconómica y política la excluye del acceso al consumo y disfrute de bienes y servicios, y de la participación en asuntos políticos."

La relación entre las variables socioeconómicas Salud y Educación han sido estudiadas a lo largo del tiempo. Ross \& Wu (1995) señalaba esta importancia, indicando que aquellos individuos que tuvieron acceso a un nivel determinado de estudios tienden a llevar una vida económica estable y por ende a una mejor calidad de vida (en sentido de salud) con respecto de aquellos que no la tuvieron. Por lo tanto, es de suponerse que el acceso a los servicios de salud a los ciudadanos desde que comienzan su vida educativa se compensará en un mejor bienestar social y por ende el desarrollo de capital humano implicará positivamente en el desarrollo económico. En este sentido, en México existen diversos grupos sociales, siendo los más vulnerables aquellos que residen en zonas rurales o agrupamientos indígenas (Ochoa Nogales et al., 2018) específicamente los menores de edad. Si bien es cierto que existen programas gubernamentales para el desarrollo de estas zonas y de apoyo a sus habitantes, también es cierto que el nivel alcanzado no ha contribuido a un desarrollo sustentable debido a la falta de comunicación pueblo-gobierno (Fernández, 2018). Para este estudio y considerando los apuntes anteriores, podemos establecer la relación que existe entre el acceso a los servicios básicos de salud específicamente en nuestro plano de estudio que es la región del altiplano potosino mediante los siguientes supuestos:

\footnotetext{
${ }^{6}$ Se utilizaron los datos presentando por el PNUD en el "Índice de desarrollo humano municipal en México: una nueva metodología", para calcular los ingresos per cápita anual de los municipios que integran a la región del altiplano, mismos que alcanzan los 7,232.5 dólares y que resultan menores al promedio estatal que es de 7,325.9 dólares.
} 


$$
\begin{gathered}
\text { Educación básica }=f(\text { Salud }) \\
\text { Educación Superior }=f(\text { Educación Básica,Salud })
\end{gathered}
$$

Considerando la suposición anterior, también podemos relacionar una variable socioeconómica que ha tenido impacto sobre todo en las regiones más vulnerables, la Marginación. En este sentido, consideramos que el poco acceso a los servicios de salud y educación, llámese básica y superior implicará un aumento en el índice de marginación. En el estudio sobre el Índice Absoluto de Marginación se mencionan los principales indicadores que influyen en la marginación entre ellos la Educación (Almejo Hernández et al., 2013). A su vez en (Ramos Soto et al., 2018), se estudian los principales factores que tienen implicación en el índice de marginación, pobreza y desigualdad encontrando que la falta de accesos a servicios básicos públicos (como pueden ser educación, salud, etc.)son disparadores de la misma. De acuerdo con lo anterior, podemos señalar el siguiente supuesto:

$$
\text { Marginación }=f(\text { Educación Superior, Salud })
$$

Las expresiones anteriores demuestran simplemente la relación entre las variables, sin embargo, podemos definirlas mediante una función lineal expresando nuestras hipótesis de la siguiente manera:

$$
\begin{gathered}
\text { Educación básica }=\beta_{1} \text { Salud }+\varepsilon \\
\text { Educación Superior }=\beta_{2} \text { Educación Básica }+\beta_{3} \text { Salud }+\varepsilon \\
\text { Marginación }=\beta_{4} \text { Educación Superior }+\beta_{5} \text { Salud }+\varepsilon
\end{gathered}
$$

Se puede argumentar entonces que existe una relación causal directa entre la variable Salud con las variables Educación Básica y Media Superior y a su vez una relación directa entre la variable Salud y la Marginación.

En un segundo aspecto, la migración como se señala en Rojas et al. (2011) se considera como el "desplazamiento geográfico de individuos o grupos, generalmente por causas económicas o sociales"7 , esta definición empata con nuestros objetivos de estudio ya que podemos identificar la variable migración como un indicador económico y social con implicaciones a partir del sector educativo y a su vez de la marginación.

En este mismo sentido, la migración es un factor fundamental para explicar el desarrollo económico. Ramos Soto et al., (2018) señalan que la migración es una fuente de desigualdad económica, para Hildebrandt \& McKenzie (2005) la migración contribuye a que las poblaciones más vulnerables (áreas rurales) tengan menos accesos a los servicios de salud, y otros servicios

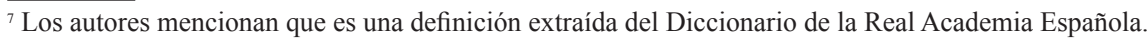


básicos como la educación. Sin embargo, la consecuencia negativa de esta relación puede verse opacada dependiendo de la cantidad de remezas que adquieran los hogares de migrantes (Acosta Rangel \& Caamal-Olvera, 2017), por lo que a mayor entrada de remesas por parte de los migrantes mayor accesos a los servicios públicos. Por lo tanto, podemos asumir que:

\section{Migración $=\beta_{6}$ Educación Básica $+\beta_{7}$ Educación Superior $+\beta_{8}$ Marginación $+\varepsilon(7)$}

Una vez establecido del modelo anterior, podemos pensar en implicar otra serie de variables que influyen al desarrollo económico de una entidad. En primera instancia las variables Marginación y Educación influyen en el desarrollo económico, como se mencionó anteriormente y en el desarrollo del modelo se optó por establecer una relación causal entre Educación Superior y Desarrollo Económico, en el entendido de que el nivel educativo también influye; es decir, si es más alto el nivel de educación en una región el desarrollo económico de ésta aumentará paulatinamente y el nivel de Marginación se reducirá en el mismo sentido, por lo que podemos sugerir que existe una relación causal entre estas dos variables, tal como:

$$
\text { Desarrollo Económico }=\beta_{8} \text { Marginación }+\beta_{9} \text { Educación Superior }+\varepsilon
$$

Como ya se mencionó, si la población asentada en una región tiene pocas oportunidades de educación y por ende limitados espacios laborales bien remunerados esto generará marginación, lo que no sólo se establecería esta brecha social negativa, sino que además significaría una movilidad poblacional en potencia; es decir, una población migrante en busca de mejores oportunidades.

\section{Análisis de los resultados}

Para realizar el contraste entre las variables del modelo es necesario establecer un análisis estadístico para obtener el valor de la regresión y su coeficiente de determinación, lo que nos permitirá realizar una representación de la realidad del objeto de investigación. Esto es, establecer la relación existente entre las variables latentes y las variables manifiestas del modelo propuesto.

Para este tipo de metodología es muy importante la recaudación de datos que confirmen la calidad del modelo y de los instrumentos de medición; en este aspecto se pueden referenciar dos tipos de medición clásicas: la confiabilidad y la validez de cada uno de los elementos y del modelo en general. Por ejemplo, Arnold et al. (2006) señalan que "La confiabilidad apunta a la eliminación de las distorsiones contingentes en la aplicación del instrumento (desde la "presencia" del encuestador, hasta el contexto de esta, pero sobre todo a la calidad muestral) 
y la validez, como relación de correspondencia entre la medición y lo medido." En relación con ello, Yong Varela (2004) señala la aplicación común en trabajos de investigación cuantitativa dos técnicas estadísticas para el análisis de datos. El primero de ellos se relaciona con la descripción y organización de los datos de tal forma que permitan al investigador "describir" la relación de las variables de investigación. La segunda técnica evalúa la inferencia del comportamiento de las variables de investigación con respecto a una muestra poblacional, este método permitirá al investigador evaluar la consistencia de un modelo e hipótesis de estudio.

Las relaciones causales entre diversas variables. Una técnica estadística para evaluar este tipo de relaciones entre variables son las regresiones múltiples mediante un análisis de rutas. Streiner (2005) señala que este tipo de instrumento permite revisar y comparar distintos modelos (complejos), sin embargo, no pueden utilizarse para establecer una causalidad o para determinar si un modelo es correcto; tan sólo consolidar la consistencia de los datos con el modelo propuesto. (Chen \& Tseng, 2012) también señalan que las técnicas de ecuaciones estructuradas son técnicas multivariantes basadas en el análisis de rutas, estas técnicas permitirán al investigador determinar la validez de la investigación empírica mediante el análisis de diversos grupos de variables y su respectiva relación causa-efecto.

En diversas áreas de investigación económica y social se han puesto en práctica estos instrumentos de análisis basados en ecuaciones estructurales. Por ejemplo, se pueden mencionar dos de las técnicas más utilizadas en este contexto: Los modelos basados en covarianzas y los modelos por mínimos cuadrados parciales (Caballero Domínguez, 2006). Entre estas dos técnicas se pueden distinguir diferencias metodológicas: En el modelo basado por covarianzas maximiza la varianza explicada de las variables latentes endógenas mediante la estimación de las relaciones de los modelos parciales en una secuencia iterativa de mínimo cuadrados ordinarios; por otro lado, los modelos basados en mínimos cuadrados parciales suelen estimar los parámetros del modelo de manera que se reduce al mínimo la discrepancia entre las matrices de covarianza y de la muestra (Joe F. Hair, Sarstedt, et al., 2011, p. 415).

La técnica PLS se caracteriza por su capacidad para analizar modelos multivariables basados en regresión lineal con altos grados de dimensionalidad, multicolinealidad y pocas observaciones. Como en todo proyecto de investigación, la selección del instrumento de análisis dependerá de los objetivos y contexto de la misma (Hair Jr et al., 2014), por lo que para este trabajo de investigación y por la complejidad del modelo que se está desarrollando adoptaremos el modelo de mínimos cuadrados parciales (PLS) para el análisis y evaluación de la consistencia del modelo. El análisis en este tipo de estudios consta principalmente de dos fases: En la primera fase se analiza la fiabilidad de los constructos y la segunda la validación de las hipótesis de estudio a través de PLS-SEM, mismos que se describirán más adelante. Para llevar a cabo los cálculos del algoritmo PLS-SEM, pruebas T y las relevancias predictivas se utilizó el software informático de modelado de SEM: SmartPLS v2.0 (Cheung 
\& Vogel, 2013). Para el análisis descriptivo y gráficos en general se hizo uso de la aplicación ofimática Microsoft Excel 2016.

Para evitar el error estadístico de tipo II, una metodología para determinar el tamaño de la muestra sugerido en (Ringle et al., 2012) es el análisis de potencia estadística, para lo cual se utilizó el software estadístico G-Power con un tamaño medio $\mathrm{f}^{2}=0.15$ y asignando como el constructo del modelo propuesto "Migración” con 3 relaciones causales (Educación Básica, Educación Superior, Marginación) para un valor óptimo de 95\% en el nivel de confianza, valor aceptable en el área de ciencias sociales (Joseph F. Hair, Sarstedt, et al., 2012), lo que estableció como un tamaño muestral mínimo de 119 elementos tal como se puede visualizar en la Tabla 1.

Tabla 1

Muestreo mínimo para un Análisis de poder de 95\%

\begin{tabular}{lll}
\hline f TESTS - LINEAR MULTIPLE REGRESSION: FIXED MODEL, R² DEVIATION FROM ZERO \\
\hline ANALYSIS: & A PRIORI: COMPUTE REQUIRED SAMPLE SIZE & $=0.15$ \\
INPUT: & EFFECT SIZE F ${ }^{2}$ & $=0.05$ \\
& A ERR PROB & $=0.95$ \\
POWER (1-B ERR PROB) & $=3$ \\
NUMBER OF PREDICTORS & $=17.8500$ \\
OUTPUT: & NONCENTRALITY PARAMETER $\Lambda$ & $=2.6835$ \\
& CRITICAL F & $=3$ \\
NUMERATOR DF & $=115$ \\
DENOMINATOR DF & $=119$ \\
TOTAL SAMPLE SIZE & $=0.9510$ \\
\hline
\end{tabular}

Fuente: Elaboración propia con relación a los resultados de G-Power.

\section{Medición del modelo}

Los modelos de análisis de rutas deben evaluarse de acuerdo con su modalidad causal; es decir, la forma en cómo se considerarán las relaciones entre las diversas variables del modelo. Para efectos de esta investigación y por las características del modelo propuesto, las relaciones entre sus variables serán consideradas reflexivas. Siguiendo a (Roldán \& Sánchez-Franco, 2012), quienes recomiendan utilizar como metodología para evaluar este tipo de relaciones procedimientos de medición, la validación convergente, discriminante y la evaluación estructural del modelo.

Para el análisis de validez convergente se deben de considerar algunas reglas, por ejemplo 
Martinez-Torres et al. (2008) señalan que este tipo de análisis indican el grado de correlación que existen entre los elementos de una escala, mismos que deberían de mostrar una correlación fuerte. Uno de los métodos más utilizados en ciencias sociales para realizar este análisis (fiabilidad de una escala y su consistencia interna) es el alfa de Cronbach. El valor aceptable en la literatura académica para esta unidad de medida es $\alpha \geq 0.7$ y $\alpha \leq 0.9$ (Oviedo \& Arias, 2005).

Se puede observar en la Tabla 2 los valores obtenidos en el modelo para el Alfa de Cronbach son superiores a 0.7 en cada una de las variables estudiadas por lo que puede considerarse que tiene una consistencia interna aceptable. Para asegurar que el modelo sea consistente Fornell \& Larcker (1981) proponen evaluar la fiabilidad compuesta de los constructos (para el área de ciencias sociales), en este caso los valores propuestos para este tipo de análisis debe ser superior a 0.7. Además de esta medida de evaluación, se deberá medir la validez convergente a través de la Varianza Media Extraída (AVE) por cada constructo del modelo. La recomendación para esta medida es $A V E>0.5$. Para nuestro caso de estudio, todos los constructos propuestos en el modelo superan los valores antes mencionados, esto supone que existe una consistencia interna en el modelo.

Análisis validez convergente y discriminante

\begin{tabular}{|c|c|c|c|c|c|c|c|c|c|c|}
\hline & \multirow[b]{2}{*}{ AVE } & \multirow[b]{2}{*}{$\begin{array}{l}\text { FIAB. } \\
\text { COMP }\end{array}$} & \multirow[b]{2}{*}{$\mathrm{R}^{2}$} & \multirow[b]{2}{*}{$a C r$. } & \multicolumn{6}{|c|}{ Cuadro de correlaciones } \\
\hline & & & & & ECO. & $\begin{array}{l}\text { ED. } \\
\text { BÁS. }\end{array}$ & $\begin{array}{l}\text { ED. } \\
\text { MED. } \\
\text { SUP. }\end{array}$ & MRG. & MIG & SALD \\
\hline ECO. & 0.9619 & 0.987 & 0.8478 & 0.9801 & 0.9808 & & & & & \\
\hline ED. BÁS. & 0.8395 & 0.9127 & 0.5882 & 0.8102 & 0.6959 & 0.9162 & & & & \\
\hline $\begin{array}{l}\text { ED. MED. } \\
\text { SUP. }\end{array}$ & 0.8922 & 0.9431 & 0.6904 & 0.8793 & 0.9094 & 0.7701 & 0.9446 & & & \\
\hline MRG. & 0.7954 & 0.8861 & 0.1123 & 0.7434 & 0.4332 & 0.2262 & 0.3260 & 0.8919 & & \\
\hline MIG. & 0.861 & 0.9253 & 0.7251 & 0.8387 & 0.7268 & 0.8073 & 0.7898 & 0.3047 & 0.9279 & \\
\hline SALD & 0.783 & 0.9351 & 0 & 0.907 & 0.7254 & 0.7670 & 0.7909 & 0.3051 & 0.7867 & 0.8849 \\
\hline
\end{tabular}

Fuente: Elaboración propia.

La validez discriminante de los constructos en un modelo sugiere que el grado en que la variable medida no debe ser un reflejo de alguna otra variable, es decir no debe existir correlación entre ellas (Martinez-Torres et al., 2008). Para realizar esta prueba, se sugiere utilizar la raíz cuadrada de la varianza media extraída de cada constructo sobre la matriz de correlación. (Fornell \& Larcker, 1981) sugieren que el resultante de esta operación debe ser 
mayor a las correlaciones de los constructos del grupo. La Tabla 2 muestra los resultantes de esta operación para el modelo propuesto, donde la raíz cuadrada del AVE (valores en negrita) son mayores a las cargas de cada constructo del grupo. La Figura 1 muestra las cargas y los pesos de las relaciones en el modelo estructural en el software SmartPLS 2.

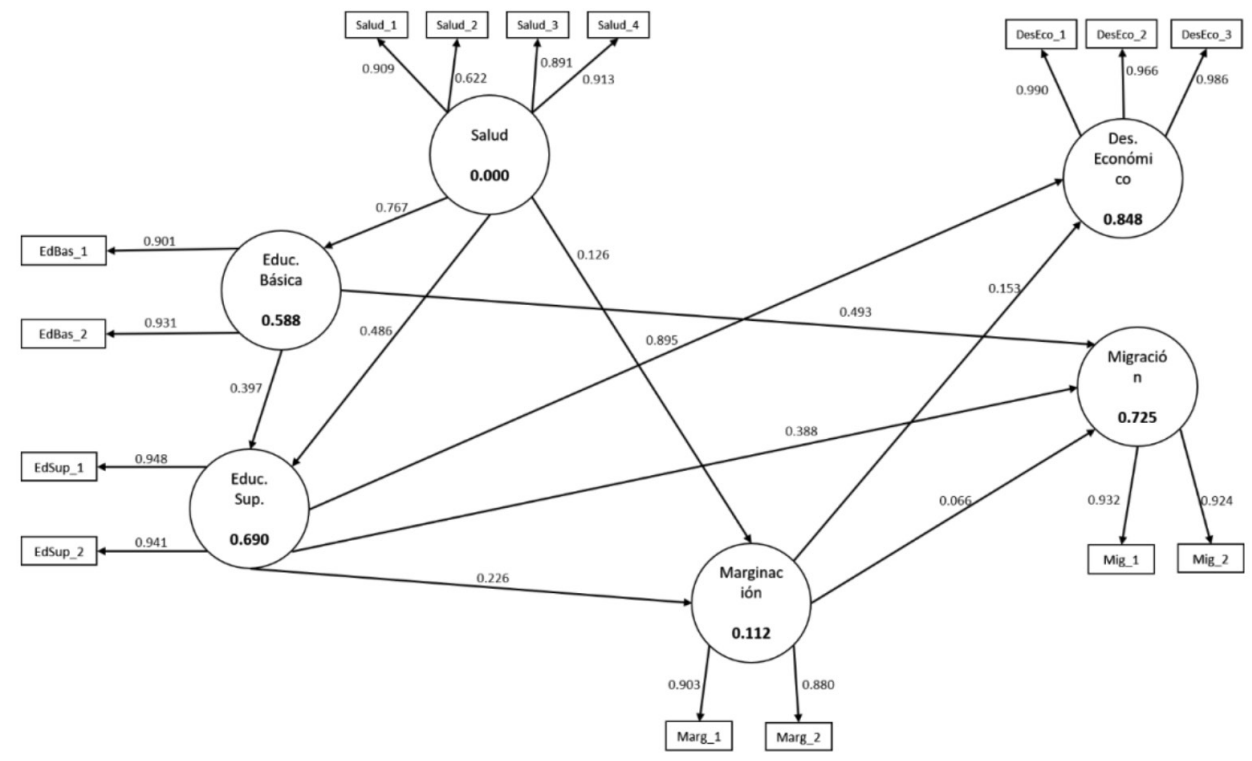

Figura 1. Resultados del Modelo estructural obtenido de SmartPLS.

Fuente: Elaboración propia.

\section{Evaluación del modelo estructural}

Como se mencionó anteriormente, en este tipo de ejercicios es importante realizar el análisis del modelo estructural mediante la significancia estadística a través del bootstrapping (remuestreo). Esta técnica consiste en realizar análisis estadísticos basados en bootstrap de un conjunto de datos que pertenecen a una muestra aleatoria reemplazando la muestra original para generar la muestra bootstarp proporcionando los errores estándar y los estadísticos $\mathrm{T}$ para la corroboración de las hipótesis planteadas. Regularmente el valor mínimo aceptado para el remuestreo es de 500 y otro aspecto de validez señala que el número de casos debe ser el mismo que el número de observaciones de la muestra original. Para este estudio nos basaremos en las recomendaciones realizadas en Joe F. Hair, Ringle, et al. (2011), en donde sugieren incrementar el número de muestras de 500 a 5 000. Para el contraste de los intervalos de confianza nos basaremos en los valores propuestos por (Roldán \& Sánchez-Franco, 2012) 
en el cual señalan que $\rho<0.05 ; \rho<0.01 ; \rho<0.001$. En relación con lo anterior, se obtendrán los estadísticos T para test de una cola definidos como:

$$
t(N-1) \rightarrow t(5000-1) \therefore t(0.05 ; 4999)=1.6451, t(0.01 ; 4999)=2.3270, t(0.001 ; 4999)=3.0902
$$

La Tabla 3 contiene los resultados del modelo estructural en la cual se puede observar que la relación que define la Marginación hacia la Migración y la relación Salud hacia Marginación no son significativas ya que no alcanzaron el criterio mínimo de la distribución para p $<0.05$ en comparación con las demás relaciones.

Tabla 3

Resultados del modelo estructural (Efectos totales). Basado en la prueba de una cola $\mathrm{t}(\mathrm{N}-1)$

\begin{tabular}{llcccc}
\hline HIP & & $\begin{array}{r}\text { EFECTO } \\
\text { ESPERADO }\end{array}$ & $\begin{array}{c}\text { COEFICIEN- } \\
\text { TE DE RUTA }\end{array}$ & $\begin{array}{c}\text { T-VALUE } \\
\text { BOOTSTRAP }\end{array}$ & SOPORTADA \\
\hline H1 & ED. BÁS $\rightarrow$ ED. MED. SUP. & + & 0.3972 & $4.2097 * * *$ & SI \\
H2 & ED. BÁS $\rightarrow$ MIG. & + & 0.4932 & $3.6527 * * *$ & SI \\
H3 & ED. MED. SUP. $\rightarrow$ ECO. & + & 0.8595 & $28.93 * * *$ & SI \\
H4 & ED. MED. SUP. $\rightarrow$ MRG. & + & 0.2262 & $1.8988^{*}$ & SI \\
H5 & ED. MED. SUP. $\rightarrow$ MIG. & + & 0.3883 & $3.1943 * * *$ & SI \\
H6 & MRG. $\rightarrow$ ECO. & + & 0.153 & $3.0671^{* *}$ & SI \\
H7 & MRG. $\rightarrow$ MIG & + & 0.0665 & 1.1248 & NS \\
H8 & SALD $\rightarrow$ ED. BÁS. & + & 0.767 & $7.3147 * * *$ & SI \\
H9 & SALD $\rightarrow$ ED. MED. SUP. & + & 0.4862 & $5.4235^{* * *}$ & SI \\
H10 & SALUD $\rightarrow$ MRG. & + & 0.1262 & 1.059 & NS \\
\hline
\end{tabular}

$* \mathrm{P}<0.05=1.64, * * \mathrm{P}<0.01=2.32, * * * \mathrm{P}<0.001-=3.092, \mathrm{NS}:$ NO SIGNIFICANTE

Fuente: Elaboración propia. Basado en la prueba de una cola t(n-1).

La evaluación del modelo estructural (además de las pruebas de hipótesis) requiere un análisis sobre los intervalos de confianza como índice de medida de incertidumbre para evitar los errores estándar derivados del remuestreo. Para calcular los intervalos de confianza del remuestreo, adoptaremos el método sugerido en Roldán \& Sánchez-Franco (2012). Este análisis sugiere realizar el análisis por percentiles con valores mínimos de $2.5 \%$ y máximos de $97.5 \%$. La Tabla 4 muestra que para este estudio las hipótesis H4, H7 y H9 no son significativas estadísticamente, se puede observar también que las relaciones Marginación $\rightarrow$ Migración (H7) y Salud $\rightarrow$ Educación Media y Superior (H9) coinciden nuevamente con respecto a los resultados de la Tabla 3. 
Tabla 4

Resultados del modelo estructural (Método de Percentiles bootstrap al 97.5\% de intervalo de confianza, $\mathrm{n}=5000$ submuestras)

\begin{tabular}{llccccc}
\hline HIP & & $\begin{array}{c}\text { EFECTO } \\
\text { ESPERADO }\end{array}$ & $\begin{array}{c}\text { COEFICIENTE } \\
\text { DE RUTA }\end{array}$ & $\begin{array}{c}\text { BAJO } \\
(2.5 \%)\end{array}$ & $\begin{array}{c}\text { ALTO } \\
(97.5 \%)\end{array}$ & SOPORTADA \\
\hline H1 & ED. BÁS $\rightarrow$ ED. MED. SUP. & + & 0.3972 & 0.2038 & 0.5715 & SI \\
H2 & ED. BÁS $\rightarrow$ MIG. & + & 0.4932 & 0.1663 & 0.6960 & SI \\
H3 & ED. MED. SUP. $\rightarrow$ ECO. & + & 0.8595 & 0.7914 & 0.9079 & SI \\
H4 & ED. MED. SUP. $\rightarrow$ MRG. & + & 0.2262 & -0.0056 & 0.4686 & NS \\
H5 & ED. MED. SUP. $\rightarrow$ MIG. & + & 0.3883 & 0.1543 & 0.6347 & SI \\
H6 & MRG. $\rightarrow$ ECO. & + & 0.153 & 0.0586 & 0.2568 & SI \\
H7 & MRG. $\rightarrow$ MIG & + & 0.0665 & -0.0297 & 0.2084 & NS \\
H8 & SALD $\rightarrow$ ED. BÁS. & + & 0.767 & 0.4941 & 0.8974 & SI \\
H9 & SALD $\rightarrow$ ED. MED. SUP. & + & 0.4862 & 0.3175 & 0.6721 & SI \\
H10 & SALUD $\rightarrow$ MRG. & + & 0.1262 & -0.0922 & 0.3806 & NS \\
\hline
\end{tabular}

Fuente: Elaboración propia.

Aunque la consideración más aceptada para explicar las variables en un modelo es la $\mathrm{R}^{2}$, se puede considerar otro criterio estadístico: la prueba de Stone-Geisrer $\left(\mathrm{Q}^{2}\right)$ sobre los constructos dependientes (Joseph F. Hair, Ringle, et al., 2012). La prueba de relevancia predictiva sugiere realizar el cálculo de validación cruzada de los componentes por redundancia y comunalidad y éstos deben ser mayores a cero. En la Tabla 5 se puede constatar que el modelo tiene una relevancia predictiva $Q^{2}>0$.

Como valor base para exponer el modelo estadísticamente se consideran los valores de , para ello adoptaremos la propuesta de (Joe F. Hair, Ringle, et al., 2011) en la cual sugieren considerar en los valores de $75 \%$ para sustancial, $50 \%$ como valor moderado y de $25 \%$ como valor explicativo débil. En la Tabla 5 se puede observar que el modelo propuesto explica en gran parte que los factores estudiados (Educación, Salud y Marginación) tienen una clara implicación en la Migración y el Desarrollo Económico en el Altiplano Potosino. El estudio sugiere que entre menos acceso tenga la población a los servicios básicos como Salud y Educación habrá un decremento en el Desarrollo Económico y por ende habrá mayor Migración. En nuestro modelo el Desarrollo Económico tiene un efecto directo con la Educación Media y Superior y la Marginación que en su conjunto explican que un $84.7 \%$ estos factores influyen a una aceleración o desaceleración en el desarrollo de la entidad; este dato podría explicar también la situación actual a nivel país en donde según el Panorama de Educación señala que el 16\% de los adultos ( 25 a 64 años de edad) cuentan con estudios de educación superior 
(Organisation for Economic Co-operation and Development, 2016). De esta misma manera el siguiente punto de estudio es la Migración de la población, en nuestro análisis la variable Migración mostró un $72.5 \%$ de explicación sobre las variables Educación Media y Superior $39.8 \%$ y Educación Básica 30.6\%, es lógico pensar que a pocas oportunidades de Educación habrá menos desarrollo y por ende la Migración se incrementará.

En el caso de las variables Educación podemos observar que ambas tienen una relación directa con la variable Salud, la cual representa 58.8\% para la educación Básica y el 69\% para la Educación Media Superior, estos datos pueden ofrecernos un panorama claro en el cual podemos sintetizar que si la población no tiene acceso a los servicios de Salud Básicos es muy probable que no culminen sus estudios Básico y de nivel Medio-Superior. Las relaciones entre Educación Media-Superior y Salud con respecto a la variable Marginación tuvieron poca explicación para este modelo con el 11.2\%, esto puede deberse a que la población no necesariamente se ve influenciada por un estatus educativo a nivel superior o los servicios de salud para considerarse marginados, sin embargo habría que considerar otros factores como la incorporación de fuentes de empleo que reduzcan la migración de la población y por ende justifique un incremento en el nivel de educación de la misma.

Tabla 5

Efectos sobre las variables endógenas

\begin{tabular}{|c|c|c|c|c|c|c|}
\hline \multicolumn{2}{|l|}{ VARIABLE E HIP. } & \multirow{2}{*}{$\begin{array}{l}\mathrm{R}^{2} \\
0.8478\end{array}$} & \multirow{2}{*}{$\begin{array}{l}\mathrm{Q}^{2} \\
0.8077\end{array}$} & \multirow[t]{2}{*}{ EFECTO } & \multirow[t]{2}{*}{$\begin{array}{l}\text { CORRELA- } \\
\text { CION }\end{array}$} & \multirow[t]{2}{*}{$\begin{array}{l}\text { VARIANZA } \\
\text { EXPLICADA } \\
\end{array}$} \\
\hline ECONOMÍA & & & & & & \\
\hline H3 & ED. MED. SUP. $\rightarrow$ ECO. & & & 0.8595 & 0.9094 & 0.7816293 \\
\hline H6 & MRG. $\rightarrow$ ECO. & & & 0.153 & 0.4332 & 0.0662796 \\
\hline $\begin{array}{l}\text { EDUCACIÓN } \\
\text { BÁSICA }\end{array}$ & & 0.5882 & 0.4733 & & & \\
\hline H8 & SALD $\rightarrow$ ED. BÁS. & & & 0.767 & 0.7670 & 0.588289 \\
\hline EDUCACIÓN & & & & & & \\
\hline MEDIA Y & & 0.6904 & 0.5799 & & & \\
\hline SUPERIOR & & & & & & \\
\hline H1 & $\begin{array}{l}\text { ED. BÁS } \rightarrow \text { ED. MED. } \\
\text { SUP. }\end{array}$ & & & 0.3972 & 0.7701 & 0.30588372 \\
\hline H9 & $\begin{array}{l}\text { SALD } \rightarrow \text { ED. MED. } \\
\text { SUP. }\end{array}$ & & & 0.4862 & 0.7909 & 0.38453558 \\
\hline MIGRACIÓN & & 0.7251 & 0.5846 & & & \\
\hline $\mathrm{H} 2$ & ED. BÁS $\rightarrow$ MIG. & & & 0.4932 & 0.8073 & 0.39816036 \\
\hline
\end{tabular}




\begin{tabular}{|c|c|c|c|c|c|c|}
\hline H5 & ED. MED. SUP. $\rightarrow$ MIG. & & & 0.3883 & 0.7898 & 0.30667934 \\
\hline H7 & MRG. $\rightarrow$ MIG & & & 0.0665 & 0.3047 & 0.02026255 \\
\hline $\begin{array}{l}\text { MARGINA- } \\
\text { CIÓN }\end{array}$ & & 0.1123 & 0.0875 & & & \\
\hline $\mathrm{H} 4$ & $\begin{array}{l}\text { ED. MED. SUP. } \rightarrow \\
\text { MRG. }\end{array}$ & & & 0.2262 & 0.3260 & 0.0737412 \\
\hline H10 & SALD $\rightarrow$ MRG. & & & 0.1262 & 0.3051 & 0.03850362 \\
\hline
\end{tabular}

Fuente: Elaboración propia.

\section{Conclusiones}

La metodología y el modelo propuestos nos permitieron establecer una relación positiva y directa entre las variables explicativas: salud, educación y marginación y las explicadas: migración y desempeño económico, lo que significa que en la medida que mejoren las condiciones socioeconómicas como la salud, o el acceso a mejores servicios básicos como el agua, la luz, el drenaje y la vivienda o las condiciones educativas,, eso tendrá efectos positivos en el desempeño económico y en la disminución de la migración, dado que existirán mejores condiciones de infraestructura y educación que permitirán que la inversión aumente y con ello la generación de empresas, empleo y producción, lo que estará cerrando un círculo virtuoso.

El altiplano potosino, es una región con poco grado de desarrollo empresarial y de negocios, lo que la somete a un círculo vicioso de bajo desarrollo social vinculado a un bajo desarrollo económico, acompañado de procesos de migración por lo que para romper con éste, es necesaria la intervención pública que promueva que mejoren las condiciones de salud, educación e infraestructura, lo que permita mejoras en las condiciones socioeconómicas al generar y aumentar el capital humano, lo que en conjunto logre cambiar la dinámica social y económica de la región.

El bajo volumen de capital humano, producto de la migración y los problemas de educación, debe estar presente en la mente de los diseñadores de políticas públicas, a la hora de buscar impulsar el crecimiento y desarrollo económico regional, ya que éste en indispensable, pero también que depende de las condiciones socioeconómicas como se probó en este trabajo.

\section{Referencias}

Acosta Rangel, R. E., \& Caamal-Olvera, C. G. (2017). Las remesas y la permanencia escolar en México. Migraciones Internacionales, 9(2), 85-111. https://doi.org/dx.doi.org/10.17428/rmi.v9i33.1333

Almejo Hernández, R., Téllez Vázquez, Y., \& López Ramírez, J. (2013). Índice absoluto de marginación 20002010 (1st ed.). Consejo Nacional de Población. Disponible en: http://www.conapo.gob.mx/es/CONAPO/Indice_Absoluto_de_Marginacion_2000_2010 Consultado:10/10/2018 
Arnold, M., Vivanco, M., Gaínza, A., Cottet, P., Canales, M., Rodríguez-Villasante, T., Ghiso, A., Asún, R., Jiménez, J., Márquez, R., Montecinos, S., \& Martinic, S. (2006). Metodologias de investigacion social Introducción a los oficios (M. Canales Cerón (ed.); Primera). Lom Ediciones.

Becker, G. S. (1964). Human Capital: A Theoretical and Empirical Analysis, with Special Reference to Education. In Notes and Queries: Vol. XVI (1st ed.). The National Bureau of Economic Research. https://doi.org/10.1093/ nq/s1-IV.92.83-a

Caballero Domínguez, A. J. (2006). SEM vs PLS: Un enfoque basado en la práctica. IV Congreso de Metodología de Encuestas, 57-66. Disponible en: https://dialnet.unirioja.es/servlet/articulo?codigo=5701265 Consultado: $10 / 10 / 2018$

Camberos, M., \& Bracamontes, J. (2009). Marginación y políticas de desarrollo social: Un análisis regional para Sonora. Problemas Del Desarrollo. Revista Latinoamericana de Economía, 38(149), 113-135. https://doi. org/10.22201/iiec.20078951e.2007.149.7666

Chen, H., \& Tseng, H. (2012). Factors that influence acceptance of web-based e-learning systems for the in-service education of junior high school teachers in Taiwan. Evaluation and Program Planning, 35(3), 398-406. https:// doi.org/10.1016/j.evalprogplan.2011.11.007

Cheung, R., \& Vogel, D. (2013). Predicting user acceptance of collaborative technologies: An extension of the technology acceptance model for e-learning. Computers \& Education, 63, 160-175. https://doi.org/10.1016/j. compedu.2012.12.003

Comité de Planeación del Desarrollo Estatal. (2016). Plan de San Luis. Periódico Oficial del Gobierno del Estado. Disponible en: https://slp.gob.mx/SECULT/pdf/Plan-Estatal-de-Desarrollo-2015-2021-(23-MAR-2016).pdf Consultado:10/10/2018

De la Torre García, R., \& Rodríguez García, C. (2014). Índice de Desarrollo Humano Municipal en México: Nueva metodología. Disponible en: https://www.mx.undp.org/content/mexico/es/home/library/poverty/idh-municipal-en-mexico--nueva-metodologia.html Consultado:10/102018

Fernández, M. I. (2018). Estrategia de inclusión Económica para la Superación de la Pobreza Rural en México (No. 247; Pobreza Rural). Disponible en: https://www.rimisp.org/wp-content/files_mf/1550604279Estrategiadeinclusióneconómicaparala.pdf Consultado: 10/10/2018

Fornell, C., \& Larcker, D. F. (1981). Evaluating Structural Equation Models with Unobservable Variables and Measurement Error. Journal of Marketing Research, 18(1), 39. https://doi.org/10.2307/3151312

Foro Consultivo Científico y Tecnológico. (2012). San Luis Potosí. Diagnóstico en Ciencia, Tecnología e Innovación. Foro Consultivo Científico y Tecnológico, AC. Disponible en: http://www.foroconsultivo.org.mx/libros_editados/diagnosticos3/san_luis_potosi.pdf Consultado:16/10/2018

Fujita, M., Krugman, P., \& Venables, A. J. (1999). The Spatial Economy. The MIT Press. https://doi.org/10.7551/ mitpress/6389.001.0001

Garduño Rivera, R. (2014). La apertura comercial y su efectoen la distribución regionalde México. Trimestre Economico, 81(322), 413-439. https://doi.org/10.20430/ete.v81i322.118

González Rivas, M. (2007). The effects of trade openness on regional inequality in Mexico. The Annals of Regional Science, 41(3), 545-561. https://doi.org/10.1007/s00168-006-0099-x

Hair, Joe F., Ringle, C. M., \& Sarstedt, M. (2011). PLS-SEM: Indeed a Silver Bullet. The Journal of Marketing Theory and Practice, 19(2), 139-152. https://doi.org/10.2753/MTP1069-6679190202

Hair, Joe F., Sarstedt, M., Ringle, C. M., \& Mena, J. A. (2011). An assessment of the use of partial least squares structural equation modeling in marketing research. Journal of the Academy of Marketing Science, 40(3), 414-433. https://doi.org/10.1007/s11747-011-0261-6

Hair, Joseph F., Ringle, C. M., \& Sarstedt, M. (2012). Partial Least Squares: The Better Approach to Structural Equation Modeling? Long Range Planning, 45(5-6), 312-319. https://doi.org/10.1016/j.lrp.2012.09.011

Hair, Joseph F., Sarstedt, M., Pieper, T. M., \& Ringle, C. M. (2012). The Use of Partial Least Squares Structural Equation Modeling in Strategic Management Research: A Review of Past Practices and Recommendations for Future Applications. Long Range Planning, 45(5-6), 320-340. https://doi.org/10.1016/j.lrp.2012.09.008 
Hair Jr, J. F., Sarstedt, M., Hopkins, L., \& Kuppelwieser, V. G. (2014). Partial least squares structural equation modeling (PLS-SEM): An emerging tool in business research. European Business Review, 26(2), 106-121. https:// doi.org/10.1108/EBR-10-2013-0128

Hildebrandt, N., \& McKenzie, D. J. (2005). The Effects of Migration on Child Health in Mexico. Economía, 6(1), 257-289. https://doi.org/10.1353/eco.2006.0009

Instituto Mexicano para la Competitividad. (2016). Índice De Competitividad Estatal 2016. Un puente entre dos Méxicos. Disponible en: https://imco.org.mx/competitividad/indice-de-competitividad-estatal-2016/ Consultado: $12 / 10 / 2018$

Kaldor, N. (1984). Causas del lento ritmo de crecimiento del reino unido. Investigación Económica, 43(167), 9-27. Disponible en: http://www.jstor.org/stable/42779413 Consultado: 14/11/2018

Krugman, P., \& Elizondo, R. L. (1996). Trade policy and the Third World metropolis. Journal of Development Economics, 49(1), 137-150. https://doi.org/10.1016/0304-3878(95)00055-0

Leyva López, S., \& Cárdenas Almagro, A. (2002). Economía de la educación: capital humano y rendimiento educativo. Análisis Económico, XVII(36), 79-106. Disponible en: http://www.redalyc.org/pdf/413/41303603.pdf Consultado: 14/11/2018

López-Rodríguez, J., Faíña, J. A., \& López-Rodríguez, J. (2007). Human Capital Accumulation and Geography: Empirical Evidence from the European Union. Regional Studies, 41(2), 217-234. https://doi. org/10.1080/00343400601108440

Martinez-Torres, M. R., Toral Marin, S. L., Barrero Garcia, F., Gallardo Vazquez, S., Arias Oliva, M., \& Torres, T. (2008). A technological acceptance of e-learning tools used in practical and laboratory teaching, according to the European higher education area. Behaviour \& Information Technology, 27(6), 495-505. https://doi. org/10.1080/01449290600958965

Mayer-Foulkes, D. (2008). The Human Development Trap in Mexico. World Development, 36(5), 775-796. https:// doi.org/10.1016/j.worlddev.2007.04.023

Ochoa Nogales, B., Camarena Gómez, B. O., Valenzuela Quintanar, A. I., \& Silveira Gramont, M. I. (2018). Condiciones socioeconómicas y de salud de grupos de población infantil que residen en localidades rurales de Sonora, México. Estudios Sociales. Revista de Alimentación Contemporánea y Desarrollo Regional, 28(51), 2-30. https://doi.org/10.24836/es.v28i51.545

Organisation for Economic Co-operation and Development. (2016). Education at a Glance 2016. OECD Publishing. https://doi.org/10.1787/eag-2016-en

Oviedo, H. C., \& Arias, A. C. (2005). Aproximación al uso del coeficiente alfa de Cronbach. Revista Colombiana de Psiquiatría, XXXIV(4), 572-580. Disponible en: http://www.redalyc.org/articulo.oa?id=80634409 Consultado:17/10/2018

Pintor Sandoval, R., Peraza Noriega, B. E., \& Heredia Trasviña, K. O. (2017). Impact of family remittances and the Prospera program on high school students in Choix, Sinaloa, Mexico. ANFORA, 24(42), 19-43. https://doi. org/10.30854/anf.v24.n42.2017.165

Ramos Soto, A. L., Cruz Carrasco, C., \& De los Santos Ascención, G. (2018). Pobreza , Marginación y Desigualdad en el Municipio de Pluma Hidalgo, Oaxaca: México. In J. Gasca Zamora \& L. Martínez Pérez (Eds.), Desigualdad Regional, Pobreza y Migración (1st ed., pp. 1-25). Universidad Nacional Autónoma de México. Disponible en: http://ru.iiec.unam.mx/id/eprint/3866 Consultado: 14/11/2018

Ringle, Sarstedt, \& Straub. (2012). Editor's Comments: A Critical Look at the Use of PLS-SEM in "MIS Quarter1y." MIS Quarterly, 36(1), iii. https://doi.org/10.2307/41410402

Rojas, G., Fritsch, R., Castro, A., Guajardo, V., Torres, P., \& Díaz, B. (2011). Trastornos mentales comunes y uso de servicios de salud en población inmigrante. Revista Medica de Chile, 139(10), 1298-1304. https://doi. org/10.4067/S0034-98872011001000008

Roldán, J. L., \& Sánchez-Franco, M. J. (2012). Variance-Based Structural Equation Modeling. In M. S. Mora, Manuel Gelman, Ovsei Steenkamp, Annette Raisinghani (Ed.), Research Methodologies, Innovations and Philosophies in Software Systems Engineering and Information Systems (pp. 193-221). IGI Global. https://doi. 
org/10.4018/978-1-4666-0179-6.ch010

Romero Rodríguez, S.I., J. J. (2010). “Informe Regional sobre Desarrollo Humano para América Latina y el Caribe 2010. Actuar sobre el futuro: romper la transmisión intergeneracional de la desigualdad." Revista de Fomento Social, 555-556. https://doi.org/10.32418/rfs.2010.259.1944

Ross, C. E., \& Wu, C. (1995). The Links Between Education and Health. American Sociological Review, 60(5), 719. https://doi.org/10.2307/2096319

Schultz, T. P. (1997). Assessing the productive benefits of nutrition and health: An integrated human capital approach. Journal of Econometrics, 77(1), 141-158. https://doi.org/10.1016/S0304-4076(96)01810-6

Schultz, T. W. (1960). Capital Formation by Education. Journal of Political Economy, 68(6), 571-583. https://doi. org/10.1086/258393

Schultz, T. W. (1961). Investment in Human Capital. In The American Economic Review (Vol. 51, Issue 1, pp. 1-17). https://doi.org/10.2307/1238690

Simón Fernández, B., Aixala Pastó, J., Giménez Esteban, G., Fabro Esteban, G., Aixalá Pasto, J., Fabro Esteban, G., Giménez Esteban, G., \& Simón Fernández, B. (2004). Determinantes del crecimiento económico. La interrelación entre el capital humano y tecnológico en Aragón. In Congreso de la Asociación Española de Ciencia Regional (XXX Reunión de Estudios Regionales) (No. 7; FUNDEAR (2003), Issue 7/04). https://doi. org/10.1192/bjp.112.483.211-a

Streiner, D. L. (2005). Finding Our Way: An Introduction to Path Analysis. The Canadian Journal of Psychiatry, 50(2), 115-122. https://doi.org/10.1177/070674370505000207

Treviño, E., Villalobos, C., \& Baeza, A. (2016). Recomendaciones de Políticas Educativas en América Latina en base al TERCE. Disponible en: http://www.unesco.org/new/fileadmin/MULTIMEDIA/FIELD/Santiago/pdf/ Recomendaciones-politicas-educativas-TERCE.pdf Consultado: 16/11/2018

Wold, H. (1975). Path Models with Latent Variables: The NIPALS Approach. Quantitative Sociology., 307-357. https://doi.org/doi.org/10.1016/B978-0-12-103950-9.50017-4

Wright, S. (1934). The Method of Path Coefficients. The Annals of Mathematical Statistics, 5(3), 161-215. https:// doi.org/10.1214/aoms/1177732676

Yong Varela, L. A. (2004). Modelo de aceptación tecnológica (TAM) para determinar los efectos de las dimensiones de cultura nacional en la aceptación de las TIC. Revista Internacional de Ciencias Sociales y Humanidades, SOCIOTAM, XIV(1), 131-171. Disponible en: https://www.redalyc.org/pdf/654/65414107.pdf Consultado: $16 / 11 / 2018$ 